\title{
Patrones de resistencia bacteriana
}

\section{en urocultivos en un hospital oncológico}

\author{
Patricia Cornejo-Juárez, MD, (') Consuelo Velásquez-Acosta, QFB, ${ }^{(2)}$ Silvia Sandoval, Enf Gral,(3) \\ Patricia Gordillo, Enf Gral, ${ }^{(3)}$ Patricia Volkow-Fernández, MD. ${ }^{(1)}$
}

\author{
Cornejo-Juárez P,Velásquez-Acosta C, Sandoval S \\ Gordillo P,Volkow-Fernández P. \\ Patrones de resistencia bacteriana \\ en urocultivos en un hospital oncológico. \\ Salud Publica Mex 2007;49:330-336.
}

\section{Resumen}

Objetivos. Describir los patrones de resistencia bacteriana en cultivos de orina en un hospital oncológico. Material y métodos. Se incluyeron las cepas obtenidas de cultivos de orina de 1998 a 2005 . Se obtuvo el porcentaje de sensibilidad para diferentes antibióticos, tras analizar por separado cepas nosocomiales y compararlas con las de la comunidad. Resultados. Se detectaron 9232 cultivos positivos (20.7\%) de 44447 muestras: gramnegativos, 78.8\%; grampositivos, 13.8\%; y levaduras, 7.4 por ciento. Escherichia coli fue el principal microorganismo identificado (4I.3\%); la resistencia en aislados nosocomiales fue mayor que en la comunidad para amikacina $(92.4$ y $97 \%)$, ceftazidima (83.I y $95.1 \%$ ) y ciprofloxacina (46.2 y 58.6\%). De igual manera, Pseudomonas aeruginosa presentó mayor resistencia para amikacina y ceftazidima en las cepas nosocomiales (55.7 y 66.6\%; y 65.5 y $84.8 \%$, respectivamente). Enterococcus resistente a vancomicina se encontró sólo en 2.5\% (3/119 aislados de E. faecium). Conclusiones. Existe una mayor resistencia bacteriana en las cepas de origen nosocomial en comparación con las cepas comunitarias. Se encontró un incremento progresivo de la resistencia para E. coli, el patógeno aislado con más frecuencia de infecciones nosocomiales y comunitarias. Es prioritario intensificar una campaña educativa para el control y uso racional de los antibióticos.
\end{abstract}

Palabras clave: resistencia bacteriana a drogas; antimicrobiano; nosocomial; orina; México

\author{
Cornejo-Juárez P,Velásquez-Acosta C, Sandoval S, \\ Gordillo P, Volkow-Fernández P. \\ Antimicrobial resistance patterns of isolates \\ from urine cultures at an oncological center. \\ Salud Publica Mex 2007;49:330-336.
}

\section{Abstract}

Objective. To describe the patterns of antimicrobial resistance of organisms isolated from urine cultures at a teaching oncological hospital for adult patients. Material and Methods. All strains obtained from urine cultures from 1998 to 2005 were included. Mean susceptibilities were obtained for each antimicrobial tested; nosocomial and communityacquired isolates were analyzed separately. Results. A total of 9232 positive urine cultures were obtained $(20.7 \%)$ from 44447 samples taken. Gram negative bacteria were reported in $78.8 \%$, Gram-positive in $13.8 \%$ and yeasts in $7.4 \%$. Escherichia coli was the most frequently isolated bacterium (4I.3\%); antimicrobial resistance was higher in nosocomial isolates than in community strains (amikacin 92.4 vs. $97 \%$, ceftazidime 83.1 vs. $95.1 \%$ and ciprofloxacin 46.2 vs. $58.6 \%$ ). Pseudomonas aeruginosa showed a greater resistance to amikacin and ceftazidime in nosocomial cultures compared to community-acquired bacterial cultures ( 55.7 vs. $66.6 \%$ and 65.5 vs. $84.8 \%$ respectively). Vancomycin-resistant enterococci were found in only $2.5 \%$ (3 of II 9 E. faecium isolates). Conclusions. Higher bacterial resistance was observed in nosocomial cultures than in community ones. Antimicrobial resistance was found to be progressively increasing for $E$. coli, the most frequent pathogen isolated both in nosocomial and community infections. We consider imperative the establishment of an intense educational campaign for the use and control of antibiotics.

Key words: bacterial drug resistance; antimicrobial; nosocomial; urine; Mexico

(I) Departamento de Infectología, Instituto Nacional de Cancerología (INCan). México, DF, México.

(2) Laboratorio de Microbiología, INCan. México, DF, México.

(3) Comité de Vigilancia y Control de Infecciones Nosocomiales, INCan. México, DF, México.

Fecha de recibido: 22 de septiembre de 2006 - Fecha de aceptado: 9 de julio de 2007

Solicitud de sobretiros: Dra. Patricia Cornejo Juárez.Departamento de Infectología. Instituto Nacional de Cancerología. Av. San Fernando 22, Col. Sección XVI, I4000 Tlalpan. México, DF Correo electrónico: patcornejo@yahoo.com 
$\mathrm{L}$ as infecciones de vías urinarias son una de las anormalidades más frecuentes y causa de incremento de la morbilidad tanto en el hospital como en la comunidad. Conocer los principales gérmenes aislados, así como los patrones de resistencia, permite determinar la variación que existe entre las diferentes especies bacterianas y orientar el inicio empírico de antibióticos con mayores elementos de acierto. ${ }^{1}$

Los patógenos urinarios más comunes han desarrollado y expresado mecanismos de resistencia adquiridos o intrínsecos, por ejemplo, las enterobacterias con producción de lactamasas beta de espectro extendido (BLEE), Pseudomonas aeruginosa y Acinetobacter spp. multirresistentes, Staphylococcus aureus resistente a meticilina y Enterococcus spp. resistentes a vancomicina. ${ }^{1,2}$

La frecuencia de aislamiento de patógenos y la resistencia bacteriana varían en grado amplio según sean las diferentes regiones geográficas, incluso entre hospitales del mismo país y ciudad. ${ }^{1}$ La Organización Mundial de la Salud ha considerado la emergencia y diseminación de la resistencia antimicrobiana como un problema prioritario y por ello desde septiembre de 2001 se instituyó una medida global para la contención de la resistencia antimicrobiana (Global Strategy for Containtment of Antimicrobial Resistance), que incluye como medida fundamental la vigilancia de la sensibilidad antimicrobiana. ${ }^{3}$ Por eso es importante publicar y dar a conocer los patrones y tendencias de sensibilidad en los diferentes hospitales del país y el mundo para aplicar o intensificar medidas estrictas de vigilancia y control del uso de los antibióticos. ${ }^{4,5}$

El objetivo de este estudio fue describir los patrones de resistencia antimicrobiana en los microorganismos aislados en cultivos de orina de enero de 1998 a diciembre de 2005, analizar por separado las muestras de pacientes con infecciones urinarias diagnosticadas como nosocomiales y distinguirlas de aquellas adquiridas en la comunidad en pacientes del Instituto Nacional de Cancerología (INCan).

\section{Material y métodos}

\section{Hospital}

El INCan es un hospital de tercer nivel, centro de referencia de toda la República mexicana de pacientes adultos con cáncer, que cuenta con 160 camas, seis camas en la unidad de terapia intensiva (UTI), una media de 7500 egresos hospitalarios, 3100 operaciones mayores y 130000 consultas al año.

\section{Protecele de trabaje}

Se realizó un estudio retrospectivo basado en los informes del laboratorio de microbiología clínica del INCan. Se incluyeron todos los cultivos de orina tomados entre enero de 1998 y diciembre de 2005.

Las muestras se inocularon en los medios agar sangre, MacConckey y Sabouraud. La identificación y sensibilidad antimicrobianas se realizaron a través de un método semiautomatizado MicroScan (DadeBehring, West Sacramento, CA, EUA). Se incluyeron los resultados de las cepas aisladas de E. coli, Klebsiella spp., Morganella morganii, Proteus spp., Enterobacter spp., Citrobacter spp., P. aeruginosa, E. faecalis, E. faecium, Candida albicans y Candida spp. Los antibióticos analizados para los gérmenes gramnegativos fueron amikacina $(\mathrm{AK})$, gentamicina $(\mathrm{GM})$, tobramicina (TOB), ceftazidima (CAZ), ceftriaxona (CAX), cefuroxima (CRM), trimetroprim/sulfametoxazol (TMP/SMX) y ciprofloxacina (CIP). Para $P$. aeruginosa se estudió además la piperacilina (PIP) y se excluyó el TMP/SMX. Para Enterococcus spp. se incluyeron ampicilina (AMP), vancomicina (VAN) y CIP.

Se consideró cultivo positivo cuando se obtuvo una cuenta mayor de $100000 \mathrm{UFC} / \mathrm{ml}$. Se determinaron cepas sensibles o resistentes de acuerdo con la concentración mínima inhibitoria, según los parámetros del Clinical and Laboratory Standards Institute (CLSI). ${ }^{6}$ Las bacterias que se encontraban en un rango intermedio se asumieron como susceptibilidad disminuida.

Las cepas de origen nosocomial se documentaron mediante el Programa de Vigilancia de Infecciones Nosocomiales, a cargo del personal adscrito al Comité de Vigilancia y Control de Infecciones Nosocomiales, quien coordina un programa permanente para la detección de episodios de infección nosocomiales con base en los criterios de la Norma Oficial SSA-26.

\section{Análisis estadístice}

Se determinó la tendencia anual de la frecuencia de resistencia de cada uno de los microorganismos especificados a los distintos antibióticos. Se obtuvo la diferencia porcentual (incremento o decremento) tras comparar las frecuencias de resistencia, al inicio y al final del tiempo del estudio. Se utilizó la prueba de la ji cuadrada y se tomó como diferencia estadísticamente significativa $p \leq 0.05$. Se analizaron en forma separada la cepas aisladas de muestras de orina procedentes de infecciones urinarias nosocomiales. 


\section{Resultados}

Durante el periodo de estudio se incluyeron 44447 cultivos de orina. Se notificaron positivos 9232 (20.7\%), de los cuales 7269 (78.8\%) fueron gramnegativos, $1275(13.8 \%)$ grampositivos y $688(7.4 \%)$ levaduras. Las muestras de origen comunitario representaron $92.7 \%(n=8556)$ y las de origen nosocomial 7.3\% ( $n=676)$ (cuadro I).

Los principales microorganismos aislados en las muestras de la comunidad fueron E. coli $(n=3$ 621, $42.3 \%)$, Klebsiella spp. $(n=425,4.9 \%)$, Proteus spp. $(n=399$, $4.7 \%)$, C. albicans $(n=297,3.5 \%)$, E. faecalis $(n=294,3.4 \%)$ y $P$. aeruginosa ( $n=175,2 \%$; figura 1$)$. Los principales microorganismos de las cepas nosocomiales fueron $E$. coli $(n=195,28.8 \%)$, C. albicans $(n=91(13.5 \%)$, P. aeruginosa $(n=57,8.4 \%)$, E. faecium $(n=46,6.8 \%)$ y E. faecalis $(n=45$, $6.6 \%$ ) (figura 2). Hasta $14.5 \%$ de las muestras de origen nosocomial se aisló en la UTI y $85.5 \%$ se aisló en pisos de hospitalización.

El germen aislado con mayor frecuencia fue $E$. coli $(41.3 \%$ del total). Las cepas de origen nosocomial presentaron menor sensibilidad a todos los antibióticos estudiados en comparación con las cepas de la comunidad (AK, 92.4 y 97\%; GM, 72.7 y $80.4 \%$; TOB,
67.1 y $77.6 \%$; CAZ, 83.1 y 95.1\%; CAX, 82.6 y 94.6\%; CRM, 62.7 y 80.2\%; CIP, 46.2 y 58.6\%; y TMP/SMX, 35 y $38 \%$ ) (figuras 3 y 4). La tendencia anual identificada mostró una disminución progresiva del porcentaje de sensibilidad para E. coli de origen hospitalario durante los últimos cuatro años en relación con todos los antibióticos estudiados.

$P$. aeruginosa presentó una diferencia estadísticamente significativa tan sólo para CAZ al comparar el promedio de sensibilidad de las cepas de la comunidad y las hospitalarias (84.8 y $65.5 \%$, respectivamente; $p=0.001$ ). Los aminoglucósidos también mostraron menor sensibilidad en las cepas nosocomiales (AK, $55.7 \mathrm{y}$ $66.6 \%$; GM, 47.5 y $52.7 \%$; TOB, 61 y $66.7 \%$; $p=n s$ ). Para $\mathrm{CIP}$, la sensibilidad promedio de las cepas nosocomiales fue mayor en comparación con las de la comunidad (71 y $64.6 \%$; $p=$ ns).

Para otras enterobacterias se encontró que las especies de Citrobacter y Enterobacter han evidenciado un incremento gradual y estadísticamente significativo de la resistencia para todos los antibióticos estudiados durante los años de observación. Citrobacter spp. presentó sensibilidad en cepas nosocomiales $(n=21)$ y comunitarias $(n=200)$ para AK de 30 y $75 \%$, CAZ de

Cuadro I

Frecuencia de EPISODIOS Y PRINCIPALES MICROORGANISMOS AISLADOS EN CULTIVOS DE ORINA. Ciudad de MéXico, enero 1998 a diciembre 2005

\begin{tabular}{|c|c|c|c|c|c|c|c|c|c|}
\hline Año de estudio & 1998 & 1999 & 2000 & 2001 & 2002 & 2003 & 2004 & 2005 & Total \\
\hline No. total cultivos & 5343 & 5716 & 6066 & 7536 & 4718 & 4867 & 4819 & 5382 & 44447 \\
\hline Cultivos positivos No. & 1048 & 1264 & 1335 & 1217 & 1158 & 1189 & 973 & 1048 & 9232 \\
\hline (\%) & $(18.2)$ & $(23.2)$ & (26) & $(23.2)$ & $(24.5)$ & $(20.1)$ & $(20.1)$ & $(19.4)$ & $(20.7)$ \\
\hline Gramnegativos - No. & 805 & 985 & 1033 & 940 & 923 & 952 & 759 & 872 & 7269 \\
\hline (\%) & (77) & (78) & (77) & (77) & (80) & (80) & (78) & $(83.2)$ & $(78.8)$ \\
\hline Grampositivos - No. & 198 & 161 & 194 & 152 & 161 & 169 & 136 & 104 & 1,275 \\
\hline (\%) & (19) & (I3) & (I5) & (I3) & (14) & (14) & (I4) & $(9.9)$ & $(13.8)$ \\
\hline Levaduras - No. & 45 & 118 & 108 & 125 & 74 & 68 & 78 & 72 & 688 \\
\hline (\%) & (4) & (9) & (8) & $(10)$ & (6) & (6) & (8) & $(6.9)$ & (7.4) \\
\hline Aislados comunitarios - No. & 944 & 1152 & 1233 & 1138 & 1098 & $113 \mid$ & 880 & 980 & 8556 \\
\hline (\%) & $(90.1)$ & (9l.I) & $(92.4)$ & (93.5) & $(94.8)$ & $(95.1)$ & $(90.5)$ & $(93.5)$ & $(92.7)$ \\
\hline Aislados nosocomiales - No. & 104 & 112 & 102 & 79 & 60 & 58 & 93 & 68 & 676 \\
\hline (\%) & $(9.9)$ & $(8.9)$ & $(7.6)$ & (6.5) & $(5.2)$ & $(4.9)$ & $(9.5)$ & $(6.5)$ & $(7.3)$ \\
\hline
\end{tabular}

Fuente: Laboratorio de Microbiología, Instituto Nacional de Cancerología 


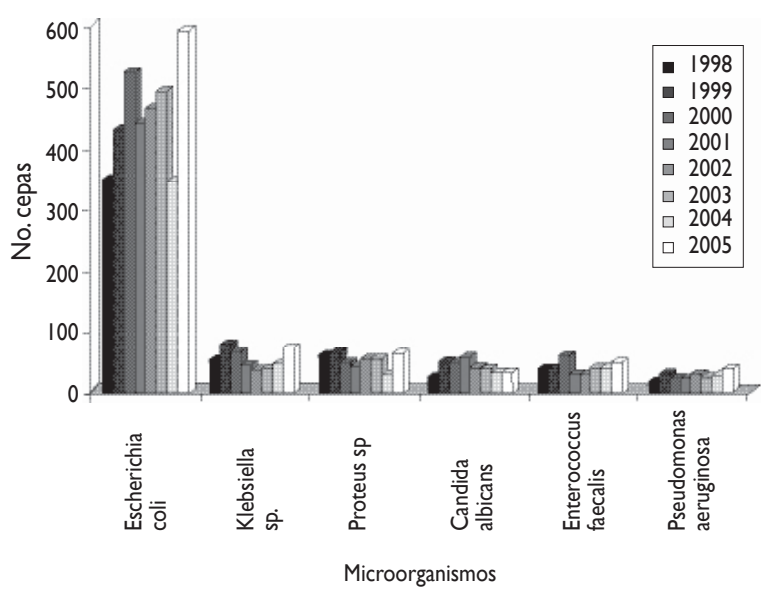

Figura I. Principales gérmenes aislados de Urocultivos de la comunidad. Ciudad de México, 1998 a 2005 $(N=52 I I)$

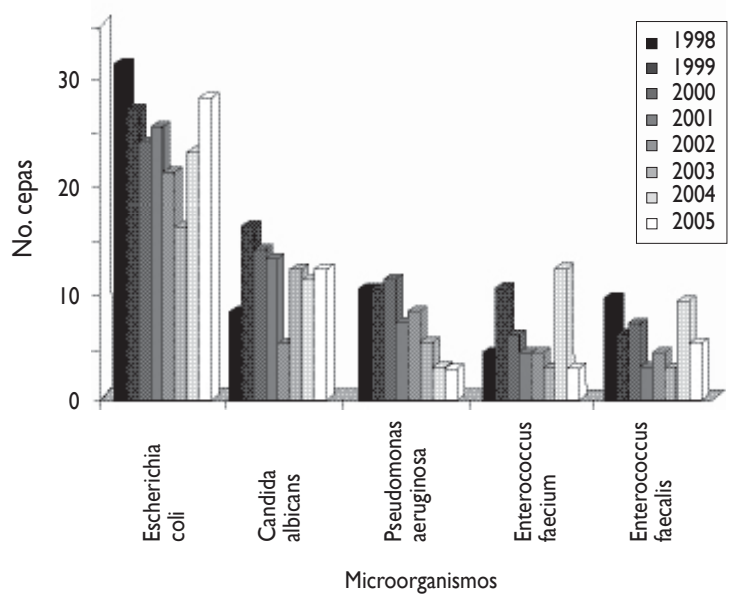

Figura 2. Principales Gérmenes aislados de URocultivos nosocomiales. INCAN. Ciudad de México, 1998 a 2005 $(\mathrm{N}=435)$

30 y $71 \%$, CIP de 28 y 58\% y TMP/SMX de 26 y 65 por ciento. Las sensibilidades para Enterobacter spp. de cepas nosocomiales $(n=19)$ y comunitarias $(n=171)$ fueron para AK de 53 y 92\%, CAZ de 46 y 85\%, CIP de 55 y $93 \%$ y $\mathrm{TMP} / \mathrm{SMX}$ de 31 y 84 por ciento.

Enterococcus faecalis fue el germen grampositivo aislado con mayor frecuencia (quinto lugar de las cepas nosocomiales y comunitarias). La sensibilidad a AMP se informó en $86.7 \%$, CIP en $48.3 \%$ y VAN en 100 por ciento. E. faecium se aisló en $1.3 \%$ del total de los cultivos $(n=119)$ y ocupó el cuarto lugar en las cepas hospitalarias. Se encontraron sólo tres cepas resistentes a VAN (2.5\%), una en 2000, otra en 2001 y una tercera en 2002, todas de origen nosocomial. Estas cepas se enviaron a un laboratorio de referencia y la resistencia se confirmó por medio de concentraciones mínimas inhibitorias

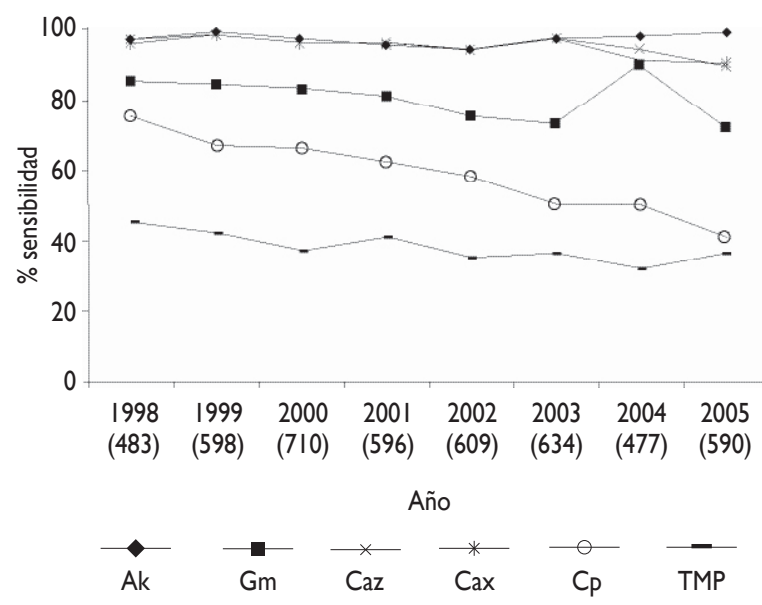

Figura 3. Susceptibilidad a antibióticos en E. coll obTENIDA DE CULTIVOS DE ORINA DE LA COMUNIDAD. CIUDAD DE MÉXICO

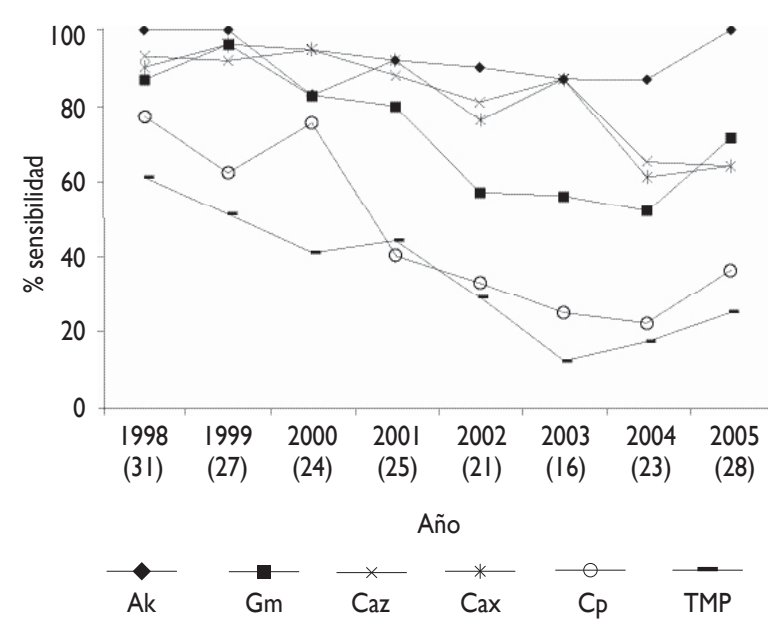

Figura 4. Susceptibilidad a antibióticos en E. coli OBTENIDA DE CULTIVOS DE ORINA NOSOCOMIALES. INCAN, Ciudad de México 
(Laboratorio de Microbiología del Instituto Nacional de Ciencias Médicas y de la Nutrición Salvador Zubirán).

Respecto de las levaduras, C. albicans fue la más aislada, al punto de ocupar el segundo lugar de las cepas nosocomiales $(13.5 \%, n=91)$ y el cuarto lugar en los aislamientos comunitarios $(3.8 \%, n=327)$.

\section{Discusión}

En los resultados obtenidos destaca el hecho de que, tal y como lo comunican estudios de vigilancia de infecciones urinarias de otras regiones del mundo, las enterobacterias son los patógenos aislados más a menudo. E. coli ocupó el primer lugar en aislamientos, si bien en un menor porcentaje (41.3\%) en comparación con los informes de la bibliografía ( 70\%). 1,5,7-10 La distribución de los gérmenes en las cepas de la comunidad incluyó a los notificados con regularidad en otros estudios, como Klebsiella spp., Proteus spp., C. albicans y Enterococcus spp. ${ }^{1}$ Asimismo, los microorganismos aislados de las muestras nosocomiales fueron aquellos habitualmente relacionados con la transmisión hospitalaria (P. aeruginosa, E. faecium, C. albicans).

Se reconoció que la sensibilidad de E. coli a fármacos utilizados con frecuencia en pacientes ambulatorios con infecciones de vías urinarias, como TMP/SMX y CIP, fue baja en grado alarmante (38 y 58.6\%, respectivamente); en consecuencia, es posible concluir que debido a esta elevada tasa de resistencia, estos fármacos quedan fuera del arsenal de la terapia empírica de las infecciones de la vía urinaria en la comunidad. Las cefalosporinas de tercera generación presentaron una sensibilidad promedio de $83 \%$ para las cepas nosocomiales, con una disminución progresiva durante los últimos cuatro años. En las cepas de la comunidad, la sensibilidad para ceftazidima y ceftriaxona se mantiene en 95 por ciento. Al comparar con los resultados de un estudio realizado en Chile, antibióticos como AK, CIP y TMP/SMX presentaron mayor sensibilidad (98.7, 94.4 y $53.4 \%$, respectivamente), mientras que CAX fue muy similar a los presentes resultados $(84.4 \%$ en Chile y $82.6 \%$ en este estudio). ${ }^{8}$ Una investigación de Brasil también notificó mayor sensibilidad a TMP/SMX (54.3\%) y CIP (92.1\%). ${ }^{5}$ Un tercer protocolo realizado en España mostró una gran sensibilidad a fármacos como CIP (88\%) y GM (97\%). ${ }^{10}$

$P$. aeruginosa presentó un decremento de la frecuencia de los aislamientos de origen nosocomial. Se aisló con una frecuencia de $2.5 \%$ y un promedio de sensibilidad en cepas hospitalarias para antibióticos potencialmente útiles contra este patógeno, como CIP, CAZ y AK, de 71.5, 65.5 y 55.8\%, respectivamente (mayor a lo comunicado en otros países).${ }^{11}$ La sensibilidad a CIP fue de $58.6 \%$ en las cepas de la comunidad y de $46 \%$ en las cepas nosocomiales en promedio durante los años de estudio, muy probablemente porque éste es un fármaco de amplia prescripción y promocionado por la industria farmacéutica para suministrarse en diferentes procesos infecciosos, incluida la infección de la vía respiratoria superior.

En un estudio multicéntrico que incluyó 210 cepas de $P$. aeruginosa procedentes de sujetos con infecciones de vías urinarias, este germen representó $7 \%$ de los aislamientos y ocupó el tercer lugar después de E. coli y Klebsiella spp. La sensibilidad a CIP se registró en $40.8 \%$ en Europa (71 cepas en 16 centros hospitalarios); 29.2\% en Norteamérica (106 cepas en 31 hospitales) y 54.5\% en Latinoamérica (33 cepas en 10 hospitales). ${ }^{7}$

La sensibilidad a CIP para otras enterobacterias estudiadas fue en promedio de $57.5 \%$ en infecciones nosocomiales y $70 \%$ en infecciones de la comunidad; por lo tanto, se confirma que CIP debe restringirse incluso en infecciones urinarias no complicadas y adquiridas en la comunidad. En individuos con infecciones urinarias complicadas o de origen nosocomial se recomienda la combinación de antimicrobianos con mayor sensibilidad, como las cefalosporinas de tercera generación junto con un aminoglucósido, y aun fármacos con mayor espectro, como aztreonam o carbapenémicos. ${ }^{12,13}$ Un estudio reciente halló que estos últimos fármacos fueron el grupo más efectivos contra patógenos gramnegativos causantes de infecciones urinarias. ${ }^{9}$ En este estudio no se evaluó de modo sistemático la sensibilidad a los agentes carbapenémicos, excepto en cepas multirresistentes (datos no mostrados).

Se identificaron tres cepas de E. faecium resistentes a VAN $(2.5 \%)$, todas de origen nosocomial, en pacientes que habían recibido antibióticos por tiempo prolongado debido a otro proceso infeccioso. El suministro de VAN en el INCan está restringido a pacientes con indicaciones específicas, los cuales se aíslan en forma estricta. ${ }^{14}$ Estos resultados son similares a los de otros protocolos efectuados en México, en los cuales el problema del enterococo resistente a la vancomicina parece ser mucho menos frecuente que lo informado en Europa y Estados Unidos. ${ }^{15-17}$

Las levaduras se han convertido en patógenos importantes en infecciones de vías urinarias. La frecuencia de aislamiento es similar a la comunicada en hospitales oncológicos y no oncológicos de Estados Unidos. ${ }^{18}$ C. albicans es la segunda causa de infección urinaria nosocomial, tal vez debido a que en los enfermos hospitalizados se conjuntan otros factores de riesgo para este tipo de infecciones, entre ellos el uso de antibióticos de amplio espectro, sondas urinarias, nutrición parenteral y estancia hospitalaria prolongada. ${ }^{19}$ 
En el INCan se emplea una política de restricción de antibióticos con base en la disponibilidad de la farmacia del hospital. Esta investigación, que complementa un estudio previo en cultivos de sangre, ${ }^{20}$ apoya el hecho de que a pesar de la restricción del uso de antimicrobianos y un programa estricto de vigilancia y control de las infecciones nosocomiales, existe una tendencia al incremento progresivo de la resistencia de las cepas aisladas en orina, así como en las de sangre, con una disminución progresiva de la resistencia a CIP durante los últimos siete años a pesar de su utilización en México durante apenas dos décadas. Destaca también que AK fue el aminoglucósido con mayor sensibilidad durante todo el periodo de estudio (se ha utilizado en los últimos 18 años en el hospital), por lo que no se justifica la disposición de rotar estos antibióticos propuesta en otros estudios. ${ }^{21,22}$

La resistencia antimicrobiana parece ser un fenómeno mucho más complejo, no sólo atribuible a un factor como el uso de uno u otro tipo de antibióticos, sino a la existencia de muchas variables aún no estudiadas y que intervienen en las directrices de prescripción, por ejemplo las medidas para el control de infecciones, los diferentes tipos de antisépticos y el movimiento de pacientes o del personal de salud de un hospital a otro; todo constituye un puente de paso de cepas multirresistentes entre diferentes hospitales.

Las principales limitaciones del estudio fueron la ausencia de estudios genéticos en las cepas aisladas, lo que impide conocer la relación entre las cepas de la comunidad y las del hospital. No se realizó en forma sistemática la sensibilidad para carbapenémicos, lo cual no permite conocer en este momento la sensibilidad real a este grupo de fármacos. Sin embargo, el comportamiento de la resistencia bacteriana en una institución como el INCan, con gran número de pacientes inmunosuprimidos, tiene particular importancia para el tratamiento de sujetos colonizados o infectados por cepas resistentes y de fácil transmisión nosocomial.

En el ámbito nacional, otros aspectos a considerar son la legislación de la prescripción de antibióticos sólo por personal médico, la promoción de una normatividad para asegurar la calidad de los medicamentos genéricos y la garantía y verificación por las instancias adecuadas de la bioequivalencia y biodisponibilidad. Es necesario intensificar la educación de ganaderos, agricultores y veterinarios para la utilización correcta de antibióticos y evitar su uso con fines no terapéuticos, como promover el crecimiento del ganado vacuno o la industria avícola. ${ }^{9,23,24}$

Todas estas medidas son indispensables para asegurar el uso adecuado y racional de los antibióticos, lo cual permitirá combatir el problema de la emergencia y diseminación de la multirresistencia antimicrobiana.

\section{Referencias}

I. Turnidge J, Bell J, Biedenbach DJ, Jones RN. Pathogen occurrence and antimicrobial resistance trends among urinary tract infection isolates in the Asia-Western pacific region: report form the SENTRY Antimicrobial Surveillance Program, 1998-1999. Int JAntimicrob Agents 2002;20:10-17. 2. Jones RN. Resistance patterns among nosocomial pathogens: trends over the past few years. Chest 2001; I 9(suppl 2):397S-404S.

3. World Health Organization-Antimicrobial resistance. Fact sheet $N^{\circ} 194$. Revised January 2002. [Consultado 19 agosto 2006]. Disponible en: www. who.int/mediacentre/factsheets/fs I 94/en/print.html.

4. Goldmann DA, Huskins WC. Control of nosocomial antimicrobialresistant bacteria: a strategy priority for hospitals worldwide. Clin Infect Dis 1997;24(suppl I):SI39-SI45.

5. Oplustil CP, Nunes R, Mendes C, RESISTNET Group. Multicenter evaluation of resistance patterns of Klebsiella pneumoniae, Escherichia coli, Salmonella spp and Shigella spp isolated from clinical specimens in Brazil. RESISTNET Surveillance Program. Braz J Infect Dis 200 I;5:8-I2. 6. National Committee for Clinical Laboratory Standards. Performance standards for antimicrobial susceptibility testing for bacteria that grow aerobically. (Eleventh Informational Supplement). Wayne (PA): NCCLS; 200I. Document MI00-SII.

7. Jones RN, Beach ML, Pfaller MA. Spectrum and activity of three contemporary fluoroquinolones tested against Pseudomonas aeruginosa isolates from urinary tract infections in the SENTRY Antimicrobial Surveillance Program (Europe and the Americas; 2000): more alike than different! Diagn Microbiol Infect Dis 200I;4I:I6I-3.

8.Valdivieso F,Trucco O, PradoV, Díaz MC, Ojeda A, Grupo PRONARES Antimicrobial resistance of agents causing urinary tract infections in II Chilean hospitals. PRONARES project. Rev Med Chil 1999;127:1033-40. 9. Savas L, Guvel S, Orlen Y, Savas N, Duran N. Nosocomial urinary tract infections: micro-organisms, antibiotic sensitivies and risk factors. West Indian Med J 2006;55:188-193.

I0.Vallano A, Rodríguez D, Barcelo ME, López A, Cano A, Vinado B, et al. Antimicrobial susceptibility of uropathogens and outcome following antibiotic treatment for urinary tract infections in primary healthcare. Enferm Infecc Microbiol Clin 2006;24:4I8-25.

I I. Carmeli Y,Troillet N, Eliopoulos GM, Samore MH. Emergence of antibiotic-resistant pseudomonas aeruginosa: comparison of risks associated with different antipseudomonal agents. Antimicrob Agents Chemother 1999;43:1379-82.

12. Patterson JE. Hospital-acquired infections: realities of risk and resistance. Chest 200I; I 9(suppl 2):426S-430S.

I3. Fridkin SK, Edwards JR, Tenover FC, Gaynes RP, McGowan JEJr. Intensive Care Antimicrobial Resistance Epidemiology (ICARE) Project: National Nosocomial Infections Surveillance (NNIS) System Hospitals. Antimicrobial resistance prevalence rates in hospital antibiograms reflect prevalence rates among pathogens associated with hospital-acquired infections. Clin Infect Dis 200I;33:324-30.

14. Recommendations for preventing the spread of vancomycin resistance recommendations of the hospital infection control practices advisory committee (HICPAC). MMWR I995;44(RR I2): I I 3.

I5. Sifuentes-Osornio J, Ponce de León A, Muñoz-Trejo T,Villalobos-Zapata Y, Ontiveros-Rodríguez C, Gómez-Roldán C.Antimicrobial susceptibility patterns and high-level gentamicin resistance among enterococci isolated in a Mexican tertiary care center. Rev Invest Clin 1996;48:91-6. 
16. Calderón-Jaimes E, Arredondo-García JL,Aguilar-Ituarte F, García-Roca P. In vitro antimicrobial susceptibility in clinical isolates of Enterococcus species. Salud Publica Mex 2003;45:96-I0I.

17. Goldmann DA, Weinstein RA, Wenzel RP, Tublan OC, Duma RJ, Gaynes $\mathrm{RP}$, et al. Strategies to prevent and control the emergence and spread of antimicrobial resistant microorganisms in hospitals. A challenge to hospital leadership. JAMA 1996;275:234-40.

18. Anaissie E. Opportunistic mycoses in the immunocompromised host: experience at a cancer center and review. Clin Infect Dis 1992;14 (suppl I):S43-S53.

19. Lundstrom T, Sobel J. Nosocomial candiduria: a review. Clin Infect Dis 2001;32:1602-7.

20. Cornejo-Juárez P, Velásquez-Acosta C, Díaz-González A, VolkowFernández P. Tendencia del perfil de sensibilidad antimicrobiana de los aislamientos de sangre en un hospital oncológico (1998-2003). Salud Publica Mex 2005;47:288-293.
2I. Raymond DP, Pelletier SJ, Cabtree TD, Gleason TG, Hamm LL, Pruett $T L$, et al. Impact of a rotating empiric antibiotic schedule on infectious mortality in an intensive care unit. Crit Care Med 2001;29: I I0I-I 108. 22. Landman D, Quale JM, Mayorga D, Adedeji A, Vangala K, Ravinshankar J, et al. Citywide clonal outbreak of multiresistant Acinetobacter baumanii and Pseudomonas aeruginosa in Brooklyn, NY.Arch Intern Med 2002;162:1515-1520.

23. Okeke IN, Laxminarayan R, Bhutta ZA, Duse AG, Jenkins P, O'Brien TF, et al. Antimicrobial resistance in developing countries. Part I: recent trends and current status. Lancet Infect Dis 2005;5:48I-493.

24. Okeke IN, Klugman KP, Bhutta ZA, Duse AG, Jenkins P, O'Brien TF, et al. Antimicrobial resistance in developing countries. Part II: strategies for containment. Lancet Infect Dis 2005;5:568-80. 